\title{
A comparison of digital subtraction angiography and computed tomography angiography for the diagnosis of penetrating cerebrovascular injury
}

\author{
William J. Ares, MD, ${ }^{1}$ Brian T. Jankowitz, MD, ${ }^{2}$ Daniel A. Tonetti, MD, ${ }^{3}$ Bradley A. Gross, MD, ${ }^{3}$ and \\ Ramesh Grandhi, MD4
}

\begin{abstract}
1Department of Neurological Surgery, NorthShore University Health System, Evanston, Illinois; ${ }^{2}$ Department of Neurological Surgery, Cooper University Hospital, Camden, New Jersey; ${ }^{3}$ Department of Neurological Surgery, University of Pittsburgh Medical Center, Pittsburgh, Pennsylvania; and ${ }^{4}$ Department of Neurosurgery, Clinical Neurosciences Center, University of Utah, Salt Lake City, Utah
\end{abstract}

OBJECTIVE Penetrating cerebrovascular injury (PCVI) is a subset of traumatic brain injury (TBI) comprising a broad spectrum of cerebrovascular pathology, including traumatic pseudoaneurysms, direct arterial injury, venous sinus stenosis or occlusion, and traumatic dural arteriovenous fistulas. These can result in immediate or delayed vascular injury and consequent neurological morbidity. Current TBI guidelines recommend cerebrovascular imaging for detection, but there is no consensus on the optimum modality. The aim of this retrospective cohort study was to compare CT angiography (CTA) and digital subtraction angiography (DSA) for the diagnosis of PCVI.

METHODS The records of all patients presenting to two level I trauma centers in the United States between January 2010 and July 2016 with penetrating head or neck trauma were reviewed. Only those who had undergone both CTA and DSA were included. Clinical and neuroimaging data were collected, and PCVIs were stratified using a modified Biffl grading scheme. DSA and CTA results were then compared.

RESULTS Of 312 patients with penetrating trauma over the study period, 56 patients ( $91 \%$ male, mean age 32 years) with PCVI met inclusion criteria and constituted the study cohort. The mechanism of injury was a gunshot wound in $86 \%(48 / 56)$ of patients. Twenty-four (43\%) patients had sustained an angiographically confirmed arterial or venous injury. Compared with DSA as the gold standard, CTA had a sensitivity and specificity of $72 \%$ and $63 \%$, respectively, for identifying PCVI. CTA had a positive predictive value of $61 \%$ and negative predictive value of $70 \%$. Seven patients (13\%) required immediate endovascular treatment of PCVI; in $3(43 \%)$ of these patients, the injury was not identified on CTA. Twenty-two patients (39\%) underwent delayed DSA an average of 25 days after injury; $2(9 \%)$ of these patients were found to harbor new pathological conditions requiring treatment.

CONCLUSIONS In this retrospective analysis of PCVI at two large trauma centers, CTA demonstrated low sensitivity, specificity, and positive and negative predictive values for the diagnosis of PCVI. These findings suggest that DSA provides better accuracy than CTA in the diagnosis of both immediate and delayed PCVI and should be considered for patients experiencing penetrating head or neck trauma.

https://thejns.org/doi/abs/10.3171/2019.8.FOCUS19495

KEYWORDS diagnostic angiography; digital subtraction angiography; computed tomography angiography; penetrating cerebrovascular injury; penetrating neurotrauma; aneurysm; dural fistula; traumatic brain injury

$\mathrm{D}$ ATA from wartime and civilian injuries suggest that the incidence of arterial vascular injury after penetrating head and neck trauma is between $3 \%$ and $42 \% .^{5}$ However, the significant mortality associated with penetrating craniocervical neurotrauma likely results in underestimating the true incidence of arterial injuries in this patient population. To date, the predominant focus in the literature on penetrating cerebrovascular injury (PCVI) has been the diagnosis and treatment of traumatic intracranial aneurysms and the possibility of resultant

ABBREVIATIONS AUC = area under the curve; $\mathrm{BCVI}=$ blunt cerebrovascular injury; $\mathrm{CCA}=$ common carotid artery; $\mathrm{CTA}=\mathrm{CT}$ angiography; $\mathrm{DSA}=$ digital subtraction angiography; ECA = external carotid artery; ICA = internal carotid artery; NPV = negative predictive value; PCVI = penetrating cerebrovascular injury; PPV = positive predictive value; UPMC = University of Pittsburgh Medical Center; UTHSA = University of Texas Health San Antonio; VA = vertebral artery.

SUBMITTED June 19, 2019. ACCEPTED August 28, 2019.

INCLUDE WHEN CITING DOI: 10.3171/2019.8.FOCUS19495. 
subarachnoid hemorrhage. However, other arterial and venous injuries have been described as sources of secondary brain fistulas, arterial dissection, dural sinus thrombosis, and injuries to the external carotid artery (ECA) branches. ${ }^{2,5-8}$ The Brain Trauma Foundation guidelines for penetrating neurotrauma state that vascular imaging should be considered in these patients (level IV evidence); however, the modality of neuroimaging is not specified..$^{14}$ The guidelines suggest that CT angiography (CTA), which has been shown to be similar in accuracy to catheter-based digital subtraction angiography (DSA) in diagnosing nontraumatic aneurysms, would be preferable to DSA if it offers results of equal accuracy. ${ }^{12}$ Here, we aim to compare the use of CTA with DSA for the diagnosis of cerebrovascular injury after penetrating neurotrauma.

\section{Methods}

\section{Study Design}

The institutional review boards of the University of Pittsburgh Medical Center (UPMC) and the University of Texas Health San Antonio (UTHSA) approved this study. All patient information was de-identified and analyzed in compliance with Health Insurance Portability and Accountability Act (HIPAA) regulations.

We retrospectively reviewed the records of all patients aged 18 years and older who had been diagnosed with penetrating craniocervical trauma and admitted to Presbyterian Hospital at UPMC or to University Hospital at UTHSA (both level I trauma centers) between January 2010 and July 2016. Patients who had not been screened with both CTA and DSA were excluded.

\section{Screening Protocol}

Once stabilized, admitted patients were screened for PCVI based on the presence of penetrating craniocervical trauma. CTA of the head and neck was performed using a 64-channel, multidetector CT scanner, and all CTA studies were read by board-certified neuroradiologists. CTA scans with metal artifacts were not excluded during the analysis. PCVI was defined as vascular injury to any craniocervical arteries and their major branches (i.e., common carotid artery [CCA], internal carotid artery [ICA], ECA, vertebrobasilar circulation) or to the cerebral dural venous sinuses. DSA was performed within 24 hours of CTA in all hemodynamically stable patients who had been determined to have potentially survivable injuries and diagnosed with PCVI based on CTA findings. In addition, if there was a high suspicion of PCVI despite negative CTA findings, DSA was performed within 24 hours of CTA. All DSA studies were interpreted by a fellowship-trained, attending interventional neurosurgeon or neurologist. In patients in whom catheter angiography had confirmed the presence of vessel injury, repeat vascular imaging was performed with CTA or DSA to assess for progression of the injured vessel.

\section{Data Collection}

Baseline demographic and admission clinical data included age, sex, mechanism of injury, location of injury (cervical vs cranial), and concomitant injuries associated
TABLE 1. Proposed PCVI-related modifications to Biffl criteria for $B C V I^{*}$

\begin{tabular}{cc}
\hline $\begin{array}{c}\text { Injury } \\
\text { Grade }\end{array}$ & Description \\
\hline I & $\begin{array}{c}\text { Luminal irregularity or dissection w/ } \\
\text { venous sinus occlusion or stenosis, ECA distribution } \\
\text { dissection or occlusion }\end{array}$ \\
\hline II & $\begin{array}{c}\text { Dissection or intramural hematoma w/ } \geq 25 \% \text { luminal narrow- } \\
\text { ing, intraluminal thrombus, or raised intimal flap }\end{array}$ \\
\hline III & $\begin{array}{c}\text { Pseudoaneurysm or fistula (CCA/ICA, VA, or ECA distribu- } \\
\text { tion) }\end{array}$ \\
\hline IV & CCA/ICA, or VA distribution occlusion \\
\hline V & Transection w/ free extravasation \\
\hline *Based on Biffl et al., 1999. Modifications appear in boldface type.
\end{tabular}

with the craniocervical trauma (e.g., fracture caused by projectile). Detailed information about the angiographic screenings was collected, including indication for PCVI screening and findings on initial CTA. Documentation of a true PCVI was identified through a review of subsequent DSA studies and confirmed by radiology reports noting the presence of vascular injury. Injury grades were assigned to each vascular injury using a modification of the Biffl criteria for blunt cerebrovascular injury (BCVI). ${ }^{3}$ Any follow-up vessel imaging that had been completed during the index or subsequent hospitalizations was recorded and analyzed. Complications from DSA were recorded as well, including access site hematoma requiring blood transfusion, symptomatic vessel dissection/injury, new postprocedural neurological deficit/stroke, and acute kidney injury secondary to contrast-induced nephropathy. Missing data were treated as omitted at random.

\section{Statistical Analysis}

The positive predictive value (PPV) of CTA was defined as 1 minus the proportion of DSA grade 0 , when the CTA grade was 1 or higher. The false-positive rates were compared for different CTA grades using a chi-square test. Significance was defined a priori as a $\mathrm{p}$ value $<0.05$. Analyses were performed using $\mathrm{R}$ software (version 3, R Foundation for Statistical Computing, GNU Affero General Public License for use).

\section{PCVI-Related Modification of Biffl Criteria for BCVI}

Given that there is no standardized grading scheme for PCVI, we are proposing modifications to the existing well-accepted and validated Biffl grading system of arterial BCVI. ${ }^{3}$ Our modified injury scale expands upon the existing grading system to allow for the inclusion of traumatic injuries to the major dural venous sinuses, the ECA and/or its branches, in addition to the vertebral artery (VA) and ICA (Table 1). In our experience, dissections or occlusion of ECA branches and occlusions of major dural venous sinuses rarely require angiographic or surgical intervention. Thus, we consider these to be grade I injuries because they are consistent with injuries that Biffl et al. 
TABLE 2. DSA-positive PCVIs, according to the modified Biffl grade

\begin{tabular}{ccc}
\hline & \multicolumn{2}{c}{ No. of Cases (\%) } \\
\cline { 2 - 3 } Modified Biffl Grade & Positive DSA & True-Positive CTA \\
\hline I & 10 & $5(50 \%)$ \\
\hline II & 2 & $2(100 \%)$ \\
\hline III & 7 & $4(57 \%)$ \\
\hline IV & 2 & $2(100 \%)$ \\
\hline V & 4 & $1(25 \%)$ \\
\hline
\end{tabular}

found very unlikely to progress and likely to heal with or without treatment. ${ }^{3}$ We chose to include traumatic arteriovenous fistulas secondary to PCVI as modified Biffl grade III lesions given our opinion that these are dynamic pathologies, similar to BCVI-induced arterial pseudoaneurysms, with potential for intracranial hemorrhage, especially in the setting of a high-risk fistula. These lesions clearly require close follow-up and may require upfront management at the discretion of the treating physician.

\section{Results}

\section{Study Population}

A total of 312 patients with penetrating head and/or neck trauma were admitted to the participating hospitals during the study period (Fig. 1). Of these, 162 (52\%) patients did not undergo any form of vascular imaging, usually because of clinical determination of a nonsurvivable injury (148/162 [91\%]). In a minority of patients (14/162 [9\%]), the penetrating injury was determined to be superficial and, based on clinical evaluation by an attending trauma surgeon, did not warrant further imaging. Among the remaining 150 patients, $86(57 \%)$ underwent CTA only and $8(5 \%)$ underwent DSA only. The remaining $56(37 \%)$ patients underwent both DSA and CTA and were included in the final analysis.

The study cohort consisted of $91 \%$ males (51/56) and had a mean age of 32 years. Forty-two patients $(75 \%)$ had sustained penetrating cranial injury, while 14 (25\%) had experienced penetrating cervical trauma. The predominant mechanism of injury was gunshot wound (48/56 $[86 \%])$.

\section{CTA and DSA Findings}

A total of 25 vascular injuries in 24 patients were identified by DSA. Among these were 6 injuries to venous structures, 6 injuries to ECA distribution arteries, 4 injuries to cervical or intracranial ICA distribution arteries, 5 injuries to cervical or intracranial vertebrobasilar distribution arteries, 3 dural arteriovenous fistulas, and 1 CCA injury. A breakdown of DSA-diagnosed injuries according to the modified Biffl grade can be found in Table 2 .

In the 24 patients with a positive CTA, 14 (58\%) had diagnosis confirmed by DSA and 10 (42\%) were found to have no evidence of vascular injury on DSA. Of the 32 patients with negative CTA, $9(28 \%)$ were found to have vascular injuries on DSA. In the 33 patients with positive imaging findings on CTA or catheter angiography, DSA confirmed the same modified Biffl grade PCVI in 13 patients (39\%). However, in 10 patients $(30 \%)$, DSA showed a higher-grade injury; in the remaining 10 patients $(30 \%)$, DSA demonstrated a lower-grade injury or no injury at all. The overall average modified Biffl grade for PCVI injuries diagnosed by DSA was 2.58, while that diagnosed with CTA was $1.96(\mathrm{p}=0.11)$. This disparity was smaller when comparing patients who had both positive DSA and CTA, where the average modified Biffl grade injury diagnosed by DSA was 2.6 while that diagnosed with CTA was $2.2(\mathrm{p}=0.46)$.

The sensitivity and specificity of CTA for diagnosing any PCVI was 0.72 and 0.63 , respectively, with an area

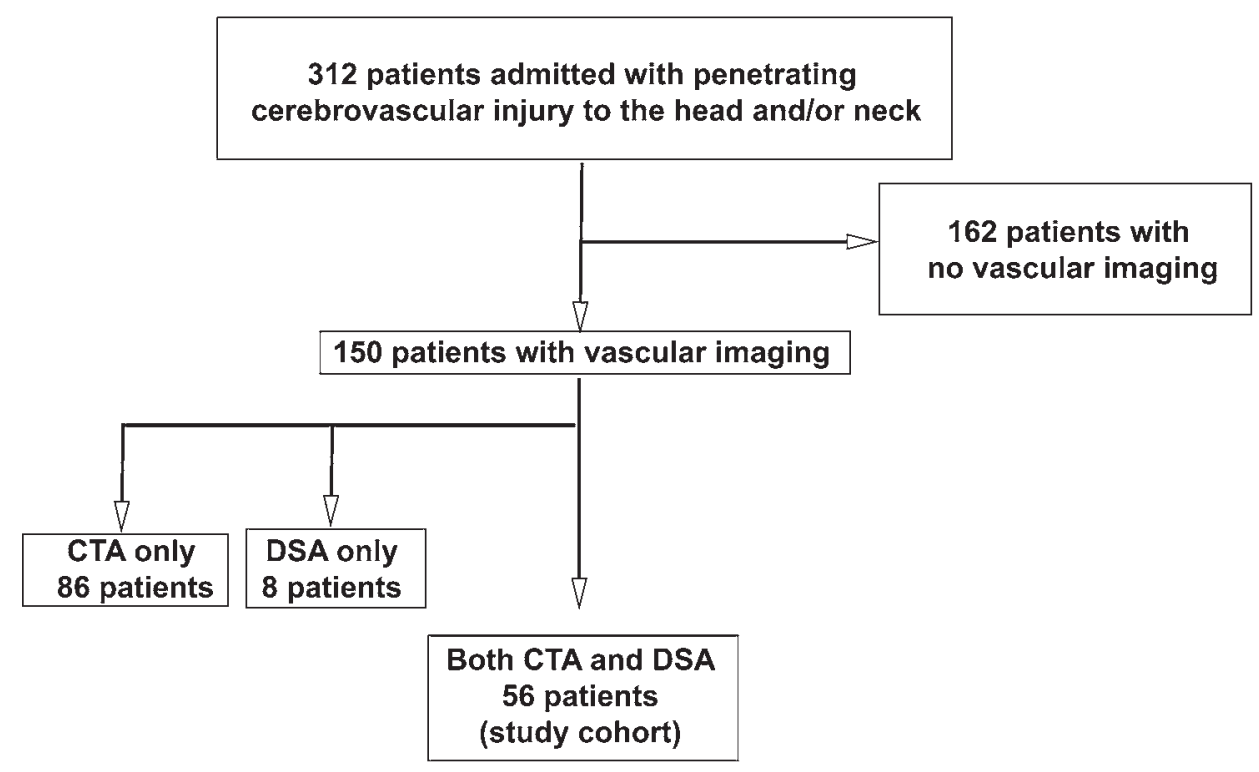

FIG. 1. Flowchart demonstrating patient accrual. 
TABLE 3. Sensitivity, specificity, and predictive values of CTA based on the component injuries

\begin{tabular}{lcccc}
\hline \multicolumn{1}{c}{ Injury } & Sensitivity & Specificity & PPV & NPV \\
\hline Any PCVI & 0.72 & 0.63 & 0.61 & 0.70 \\
\hline $\begin{array}{l}\text { Intracranial injury (ICA, pst } \\
\text { circulation, AVF) }\end{array}$ & 0.50 & 0.88 & 0.50 & 0.88 \\
\hline $\begin{array}{l}\text { Extracranial injury (CCA, cervi- } \\
\text { cal ICA, cervical VA) }\end{array}$ & 1.00 & 0.79 & 0.50 & 1.00 \\
\hline ECA injury & 0.33 & 1.00 & 1.00 & 0.85 \\
\hline Venous injury & 0.50 & 1.00 & 1.00 & 0.89 \\
\hline
\end{tabular}

$\mathrm{AVF}=$ arteriovenous fistula; $\mathrm{pst}=$ posterior.

under the curve (AUC) of 0.67 . The PPV of a positive CTA was $61 \%$ while the negative predictive value (NPV) of a negative CTA was 70\%. The PPVs of CTA for the component injuries are shown in Table 3. Shrapnel and/or metal artifact were present in $47 \%$ of CTAs that proved false positive or false negative and in $41 \%$ of CTAs that proved true positive or true negative after performing DSA ( $\mathrm{p}=$ $0.70)$.

The difference in false-positive and false-negative rates between the two centers included in our study was minimal: $34 \%$ of CTAs performed at UPMC were false positives or negatives based on confirmatory DSA, and 33\% were false positives or negatives at UTHSA.

\section{Fracture and PCVI}

Fracture patterns associated with a projectile trajectory on noncontrast head CT scans were analyzed to determine any association with PCVI (Table 4). Fractures of the cervical spine were positively associated with discovery of PCVI on DSA ( $p=0.01)$, while fractures of the frontal sinus and the orbit were negatively associated with the discovery of PCVI ( $p=0.02$ and 0.01 , respectively). No association with PCVI was found with fractures of the skull base or the calvaria or with the presence of intracranial shrapnel.

\section{Complications}

No major complications, including stroke, groin hematoma requiring blood transfusion, femoral pseudoaneurysm, or acute kidney injury, were caused by the DSA.

\section{PCVI Requiring Urgent Endovascular Management}

In this population, 7 vascular injuries were discovered via DSA that required immediate endovascular management (2 traumatic intracranial aneurysms, 1 CCA pseudoaneurysm, 4 ECA branches with active extravasation). CTA was found to accurately diagnose only $4(57 \%)$ of the 7. No complications of angiography were noted in the patient population undergoing endovascular intervention.

\section{Delayed Assessment of PCVI}

Of the 56 original patients, 25 (45\%) had repeat vascular imaging at an average of 25 days after initial angiography. Three patients underwent noninvasive imaging in the form of CTA $(n=2)$ and MRA $(n=1)$, which did not
TABLE 4. Head CT correlations with PCVI

\begin{tabular}{lccc}
\hline \multicolumn{1}{c}{ Pattern } & PCVI+ & PCVI- & p Value \\
\hline Shrapnel & $50 \%$ & $37 \%$ & 0.36 \\
\hline Calvarial fracture & $38 \%$ & $41 \%$ & 0.82 \\
\hline Facial fracture & $33 \%$ & $62 \%$ & 0.03 \\
\hline Skull base fracture & $8 \%$ & $15 \%$ & 0.42 \\
\hline Cervical fracture & $21 \%$ & $0 \%$ & 0.01 \\
\hline
\end{tabular}

Boldface type indicates statistically significant result at $p<0.05$.

demonstrate any new findings. In the 22 patients who underwent DSA follow-up, 13 had evidence of PCVI on initial angiography and 9 had previously shown no injuries. All 3 patients with venous sinus occlusions (modified Biffl grade I) attained recanalization regardless of treatment with antiplatelet agents. One patient with a modified Biffl grade II ICA dissection was found to have an improved non-flow-limiting dissection and was continued on antiplatelet monotherapy. The 3 patients with traumatic dural arteriovenous fistulas (modified Biffl grade III) demonstrated complete spontaneous resolution. One patient with a modified Biffl grade IV VA occlusion developed an associated arteriovenous fistula at the site of occlusion; in the other patient with VA occlusion, the artery remained occluded despite antiplatelet therapy. Of the 9 patients who had an initially negative DSA, one was found to have delayed formation of a distal middle cerebral artery distribution pseudoaneurysm and underwent Onyx embolization at the time of follow-up angiography. In all, on shortinterval DSA follow-up, 2 (9\%) of 22 patients were found to have pathology requiring additional treatment.

\section{Discussion}

Penetrating traumatic brain injury (TBI) is a morbid entity, with mortality after a gunshot wound to the head exceeding $90 \%$ in some studies. ${ }^{1}$ Patients who survive the initial insult may be at high risk for secondary injury if cerebrovascular injuries are not appropriately diagnosed. Official guidelines for the management of penetrating TBI do not recommend a modality for assessing cerebrovascular injury after penetrating neurotrauma; thus, the optimal modality to maximize both diagnostic yield and patient safety has been left open for debate. There has been limited investigation into the comparison between catheterbased DSA and CTA, and the few studies that have examined it have had limited generalizability because they have focused only on arterial vascular injuries and have not included CTA studies that contain metal artifacts. ${ }^{5,6}$ In this study, we aimed to compare the real-world utility of the two techniques to determine which is optimal to diagnose any penetrating neurovascular injury, including those to the ICA and ECA distributions as well as the major dural venous sinuses.

In 56 patients at two level I trauma centers who had undergone both DSA and CTA for the assessment of PCVI, we found that CTA had a sensitivity and specificity of 0.72 and 0.63 , respectively, for diagnosing any vascular injury as compared with the gold-standard DSA. This corre- 
sponded to an AUC of 0.67 , indicating a poor test. In other words, the findings of DSA changed the management of $33 \%$ of patients. This rate of change in management, while not as pronounced as the $56 \%$ seen in our comparison of CTA and DSA in BCVI, ${ }^{10}$ may be more noteworthy because many of the changes in management among the patients involved an intervention aimed at mitigating the potential of a future hemorrhagic event. Separating intracranial and cervical vascular injuries revealed that the diagnostic yield of CTA in the diagnosis of PCVI is better with cervical injuries (AUC $=0.78$ ) and demonstrably worse with intracranial injuries $(\mathrm{AUC}=0.61)$. These findings are consistent both with previous studies suggesting a limited overall sensitivity for CTA in the diagnosis of penetrating intracranial arterial injuries and with the current standard for initial evaluation of penetrating injury to the neck. ${ }^{4}$ Analysis of the noncontrast head CT findings showed that the presence of shrapnel or metal artifact did not increase the rate of false positives or false negatives on CTA, nor was the presence of shrapnel associated with an increased likelihood of the discovery of a PCVI. Additionally, facial fractures, specifically orbital and frontal sinus fractures, were significantly more likely to be associated with the absence of PCVI. Our findings corroborate those of a previous study that demonstrated the scarcity of vascular injury in the setting of frontal sinus and orbital gunshot wounds, likely because of the lack of significant vascularity in the offending trajectory. ${ }^{9}$

Nearly $10 \%$ of patients included in our study who had undergone follow-up angiography during the index hospitalization demonstrated new pathological conditions that required treatment, which underscores the importance of close vascular follow-up in patients with penetrating neurotrauma. Delayed development of vascular injuries after penetrating neurotrauma has been described previously, and the official guidelines recommend repeat angiography in these patients 2-3 weeks after presentation. ${ }^{11,14}$ In our practice, we have found that a 2 -week follow-up is sufficient to capture most, if not all, delayed injury presentations.

In summary, our study has demonstrated that DSA is an important diagnostic modality in patients with PCVI because of the low sensitivity, specificity, PPV, and NPV of CTA. Our inclusion of two different level I trauma centers, with each center demonstrating a similarly low diagnostic yield of CTA in the setting of PCVI, indicates that the results were not isolated to a single group of radiologists. In addition, delayed screening of patients with DSA is crucial among patients with PCVI given the potential for the development of delayed cerebrovascular injuries; an added benefit of performing DSA is that the interventionalist can immediately intervene on pathological conditions that require treatment.

Two principal limitations of this study are its retrospective nature and small sample size. Other limitations include our analysis of only the patient cohort that underwent both DSA and CTA, which may have under-represented the true impact and utility of DSA among patients presenting with PCVI. In addition, although we did not find the presence of shrapnel to be a statistically significant confounding variable in the diagnosis of PCVI on CTA, one may posit that in a larger series, this could be statistically significant. Importantly, the advent of new technologies such as dual-energy CT that reduces scatter artifact from metal ${ }^{13}$ and its implementation for screening patients with PCVI may represent a future direction for improving the diagnostic yield of CTA.

\section{Conclusions}

DSA is more accurate and sensitive than CTA in the diagnosis of PCVI after penetrating neurotrauma. The use of DSA can avoid the overtreatment of injuries in up to $41 \%$ of cases (CTA false positives), can avoid missing injuries in up to $28 \%$ of cases (CTA false negatives), improves recognition of cases requiring urgent endovascular management, and provides an avenue for therapeutic intervention. Given the data presented here, we believe that all patients with penetrating neurotrauma should have at least one catheter-based DSA once stabilized after their initial trauma and that consideration should be given to repeat vascular imaging within 2 weeks of injury.

\section{References}

1. Aarabi B, Tofighi B, Kufera JA, Hadley J, Ahn ES, Cooper $\mathrm{C}$, et al: Predictors of outcome in civilian gunshot wounds to the head. J Neurosurg 120:1138-1146, 2014

2. Bell RS, Vo AH, Roberts R, Wanebo J, Armonda RA: Wartime traumatic aneurysms: acute presentation, diagnosis, and multimodal treatment of 64 craniocervical arterial injuries. Neurosurgery 66:66-79, 2010

3. Biffl WL, Moore EE, Offner PJ, Brega KE, Franciose RJ, Burch JM: Blunt carotid arterial injuries: implications of a new grading scale. J Trauma 47:845-853, 1999

4. Bodanapally UK, Dreizin D, Sliker CW, Boscak AR, Reddy RP: Vascular injuries to the neck after penetrating trauma: diagnostic performance of 40- and 64-MDCT angiography. AJR Am J Roentgenol 205:866-872, 2015

5. Bodanapally UK, Saksobhavivat N, Shanmuganathan K, Aarabi B, Roy AK: Arterial injuries after penetrating brain injury in civilians: risk factors on admission head computed tomography. J Neurosurg 122:219-226, 2015

6. Bodanapally UK, Shanmuganathan K, Boscak AR, Jaffray PM, Van der Byl G, Roy AK, et al: Vascular complications of penetrating brain injury: comparison of helical CT angiography and conventional angiography. J Neurosurg 121:12751283,2014

7. Diaz-Daza O, Arraiza FJ, Barkley JM, Whigham CJ: Endovascular therapy of traumatic vascular lesions of the head and neck. Cardiovasc Intervent Radiol 26:213-221, 2003

8. Giladi O, Steinberg DM, Peleg K, Tanne D, Givon A, Grossman E, et al: Head trauma is the major risk factor for cerebral sinus-vein thrombosis. Thromb Res 137:26-29, 2016

9. Gönül E, Erdoğan E, Taşar M, Yetişer S, Akay KM, Düz B, et al: Penetrating orbitocranial gunshot injuries. Surg Neurol 63:24-31, 2005

10. Grandhi R, Weiner GM, Agarwal N, Panczykowski DM, Ares WJ, Rodriguez JS, et al: Limitations of multidetector computed tomography angiography for the diagnosis of blunt cerebrovascular injury. J Neurosurg 128:1642-1647, 2018

11. Haddad FS, Haddad GF, Taha J: Traumatic intracranial aneurysms caused by missiles: their presentation and management. Neurosurgery 28:1-7, 1991

12. International Brain Injury Association: Brain Injury Association, American Association of Neurological Surgeons, Congress of Neurological Surgeons: Part 1: Guidelines for the management of penetrating brain injury. Introduction and methodology. J Trauma 51 (2 Suppl):S3-S6, 2001 
13. Jagoda P, Schmitz D, Wagenpfeil S, Bücker A, Minko P: Comparison of metal artifact reduction in dual- and singlesource CT: a vertebral phantom study. AJR Am J Roentgenol 211:1298-1305, 2018

14. McKinney AM, Palmer CS, Truwit CL, Karagulle A, Teksam M: Detection of aneurysms by 64-section multidetector CT angiography in patients acutely suspected of having an intracranial aneurysm and comparison with digital subtraction and 3D rotational angiography. AJNR Am J Neuroradiol 29:594-602, 2008

\section{Disclosures}

Dr. Gross is a consultant for MicroVention. Dr. Jankowitz is a consultant for Medtronic. Dr. Grandhi is a consultant for Medtronic, Balt, and Cerenovus.

\section{Author Contributions}

Conception and design: Jankowitz, Ares, Grandhi. Acquisition of data: Jankowitz, Ares, Grandhi. Analysis and interpretation of data: Jankowitz, Ares, Tonetti, Grandhi. Drafting the article: Jankowitz, Ares, Grandhi. Critically revising the article: all authors. Reviewed submitted version of manuscript: all authors. Approved the final version of the manuscript on behalf of all authors: Jankowitz. Administrative/technical/material support: Tonetti.

\section{Correspondence}

Brian T. Jankowitz: Cooper Neurologic Institute, Camden, NJ. bjankowitz@gmail.com. 